\title{
Post-operative Delirium after Cardiac Surgery: Can it be Prevented?
}

\section{Sandeep Kumar Kar \\ Cardiac Anaesthesiology, Institute of Postgraduate Medical Education \& Research, Kolkata, India}

*Corresponding author: Sandeep Kumar Kar, Assistant Professor, Cardiac Anaesthesiology, Institute of Postgraduate Medical Education \& Research, Kolkata, India, Tel: 9477234900; E-mail: sndpkar@yahoo.co.in

Received date: April 25, 2016; Accepted date: April 25, 2016; Published date: April 27, 2016

Copyright: $\odot 2016$ Kar SK. This is an open-access article distributed under the terms of the Creative Commons Attribution License, which permits unrestricted use, distribution, and reproduction in any medium, provided the original author and source are credited.

Citation: Sandeep Kumar Kar (2016) Post-operative Delirium after Cardiac Surgery: Can it be Prevented?. Epilepsy J 2: 1000e107. doi:10.4172/2472-0895.1000e107

\section{Editorial}

Since 1953 Cardiopulmonary Bypass (CPB) is used during cardiac surgeries [1]. It helps in performing open heart surgeries and most of the Coronary Artery Bypass Grafting (CABG) surgeries [2]. Impetus should be given on neurological complications of CPB as they affect mortality, morbidity and quality of life [3].

Blachly and Starr used the term postcardiotomy delirium for delirium states caused after cardiac surgery. Earlier this term was used for only for on-pump cardiac surgeries, but later on this terminology of delirium included cases with delirium due to CABG too [4].

The incidence of postoperative delirium is $50-67 \%$ in post-operative cardiac patients. Delirium in post-operative patients is associated with impaired postoperative cognition, prolonged hospital stay and higher possibility of death. The similarity in features of dementia and age related changes in the brain make it very difficult to recognize this complication.

Studies have found that risk of delirium after open heart surgeries to be higher as compared to other surgeries and twice as much incidence after valve surgeries as compared to CABG. The cause of delirium after cardiac surgery is not very well known. The triggering of this situation points towards inflammation, acute stress responses and disruption of transmission of neurological pathways due to systemic inflammatory response syndrome, caused by the $\mathrm{CPB}$ process.

Many reviews have stressed on cardiopulmonary bypass and cardiac surgery as causes for postoperative cognitive effects. Postoperative delirium incidence depends on the intraoperative events mainly hemodynamic perturbations, causing cerebral perfusion pressure to fall beyond the brain's auto regulatory limits, which causes reduced cerebral perfusion. Duration of the surgical procedure is another factor, cardiopulmonary bypass time aortic cross clamp time and also the course of events during weaning from bypass, all of these cumulatively in a synergistic manner affect the postoperative cognitive status of the patients.
Postoperatively in the Intensive Care Unit (ICU) after cardiac surgery certain variables amount to higher risk of developing delirium. Length of ICU stay, duration of mechanical ventilation and low cardiac output syndrome are found to be closely related to incidence of postoperative delirium. Anemia leading to cerebral hypoxia is also found to be a risk factor in causation of delirium with increased incidence seen in patients with low haemoglobin or those requiring blood transfusions after surgery [5].

In a nutshell, Delirium after cardiac surgery is multifactorial and depends on factors such as advanced age, catabolic state, duration of $\mathrm{CPB}$ and surgery and postoperative ICU stay and mechanical ventilation. Delirium in turn prolongs recovery time and increases the possibility of death. Judicious selection of patients, aims at prevention of intraoperative and postoperative adverse events, reducing time of $\mathrm{CPB}$ and aortic cross clamp time, and decreasing the duration of the surgery will reduce the incidence of delirium after cardiac surgery and lead to better cognitive outcomes after cardiac surgery.

\section{References}

1. Gibbon JH Jr (1968) Development of the artificial heart and lung extracorporeal blood circuit. J Am Med Assoc 206: 1983-1986.

2. Heart Disease and Stroke Statistics (2005) Update. Dallas: American Heart Association.

3. Shaw PJ, Bates D, Cartlidge NEF, French JM, Heaviside D, et al. (1989) An analysis of factors predisposing to neurological injury in patients undergoing coronary bypass operations. Q J Med 72: 633-646.

4. Blachly PH (1964) Starr A: Post-cardiotomy delirium. Am J Psychiatry 121: 371-375.

5. Norkien I, Ringaitien D, Kuzminskait V, Sipylait J (2013) Incidence and Risk Factors of Early Delirium after Cardiac Surgery. Biomed Res Int 323491 . 